\title{
REVIEWS
}

\section{Cell migration promotes dynamic cellular interactions to control cerebral cortex morphogenesis}

\author{
Carla G. Silva ${ }^{1,2}$, Elise Peyre ${ }^{1,2}$ and Laurent Nguyen (i) $^{1 \text { * }}$
}

Abstract | The cerebral cortex is an evolutionarily advanced brain structure that computes higher motor, sensory and cognitive functions. Its complex organization reflects the exquisite cell migration and differentiation patterns that take place during embryogenesis. Recent evidence supports an essential role for cell migration in shaping the developing cerebral cortex via direct cellular contacts and spatially organized diffusible cues that regulate the establishment of its cytoarchitecture and function. Identifying the nature of the crosstalk between cell populations at play during brain development is key to understanding how cerebral cortical morphogenesis proceeds in health and disease.

\section{Cytoarchitectonics The cellular composition of a biological tissue. \\ Cortical wall \\ Part of the dorsal forebrain that corresponds to the presumptive cerebral cortex.}

Interneuron specification Cellular process engaging a precursor to self-autonomously acquire functional and morphological features of interneurons when placed in a neutral environment.
'GIGA-Stem Cells, University of Liège, CHU Sart Tilman, Liège, Belgium.

${ }^{2}$ These authors contributed equally: Carla G. Silva, Elise Peyre

*e-mail: Inguyen@uliege.be https://doi.org/10.1038/ s41583-019-0148-y
Cell migration controls key morphogenic events that shape the nervous system, ranging from neural tube closure to brain formation. During cerebral cortex development, cell migration is essential to set a precise temporal and spatial distribution pattern of neural cells that further engage in dynamic crosstalk to coordinate their maturation. Cerebral cortical activity relies on neural circuits formed of two main classes of neurons: excitatory projection neurons (PNs) that migrate along radial glia (RG) fibres within the cortex and inhibitory interneurons (INs) that originate from the ventral forebrain and reach the cortex along two stereotypical tangential routes ${ }^{1}$. These neuronal classes include multiple subtypes for which proper laminar positioning and balanced integration into neural networks are determinant factors for cortical function. Cortical cytoarchitectonics reflects interplays between cell extrinsic cues and intrinsic mechanisms that coordinate the migration of neurons from their birthplace to a final destination, where they assemble into functional circuits. Most of these signals are involved in the control of cytoskeletal elements and their regulators to support dynamic shape changes underlying cell motility and allocation to ad hoc cortical layers ${ }^{2}$. Migrating neurons not only receive important cues that direct their navigation and differentiation into the cortex but also influence morphogenetic events occurring in the vicinity of their migratory path. Recent reports have placed glia, and in particular microglia, the resident macrophage of the brain, as essential players for cortical morphogenesis via regulation of brain wiring and IN migration in the cortical wall. However, despite their prominent roles in cortical development, the migration pattern of the glial cells that transiently or permanently populate the cerebral cortex remains largely unexplored ${ }^{3,4}$ Here, we review the migration strategies adopted by neural cells to navigate in the cortical wall and offer perspectives for the roles of cell migration in the formation of the cerebral cortex. We also discuss how bringing together quantitative experimental analyses with mathematical modelling fosters the discovery of new mechanisms of cortical morphogenesis 5 . Furthermore, this Review sheds light on recent technological developments that advance our understanding of human cerebral cortical morphogenesis and help us decipher how cell migration deficits can interfere with this process in brain pathology.

\section{Cell migration in cortical development}

Cell migration is an important process that allows distinct cell types generated in different brain regions to settle in the cerebral cortex during embryogenesis. In mice, transient cell populations start colonizing the dorsal forebrain at embryonic day 10.5 (E10.5), and these cells guide the later migration and placement in the developing cortex of neurons generated between E11.5 and E18.5. In addition, most glial cells invade the cortical wall concurrently with neurons (FIG. 1a), with which some establish crosstalk.

\section{Cell migration strategies}

Discontinuous migration of cortical interneurons. Cortical INs (cINs) exist in different shapes and forms, and their progenitors initiate interneuron specification in the ganglionic eminences (GEs) at the onset of corticogenesis and before engaging in tangential migration ${ }^{6,7}$. Transcriptional programmes are largely conserved 

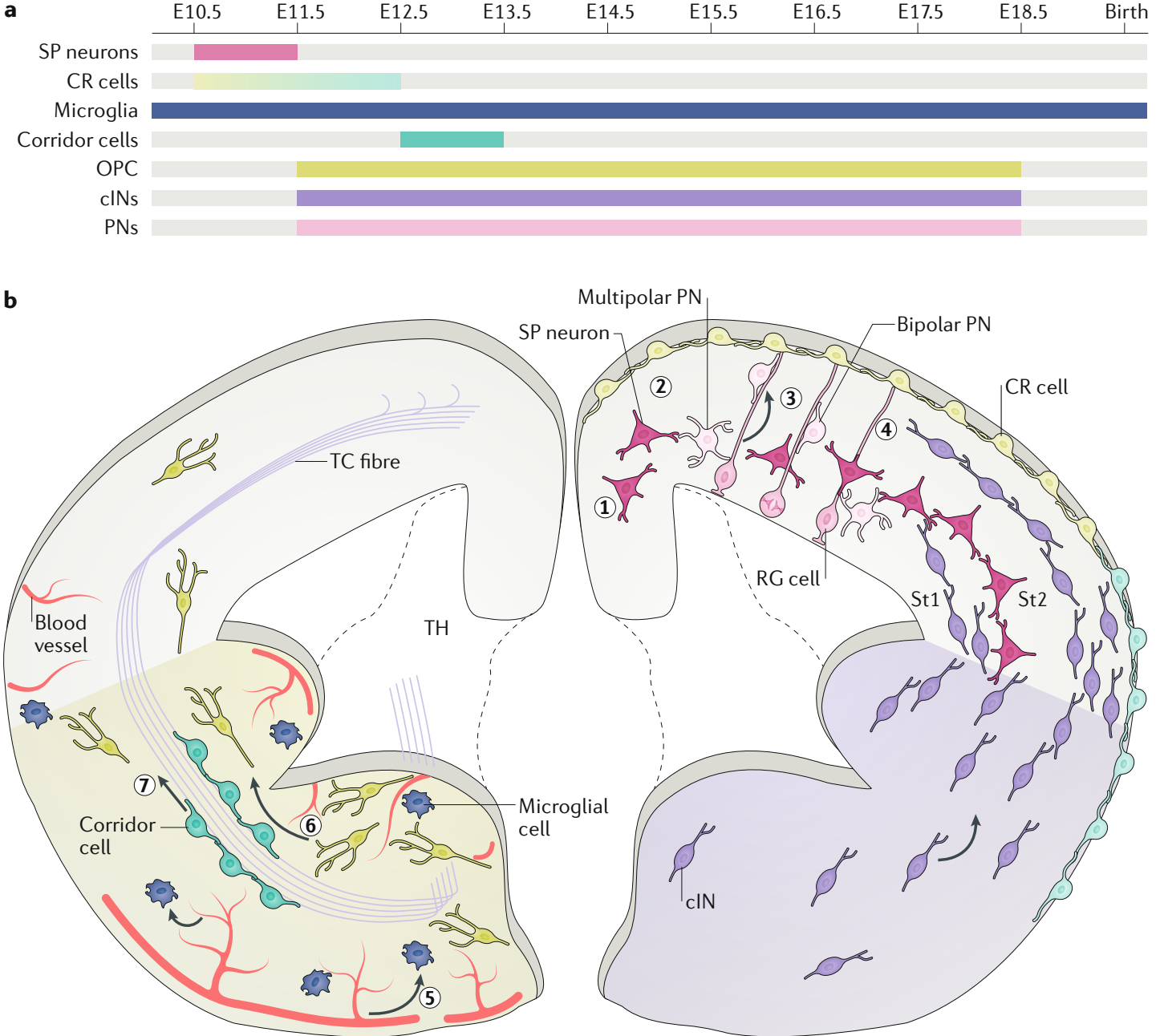

Fig. 1 | Migration strategies used by neural cells to colonize the mouse developing cerebral cortex. a |Timeline of generation and migration of cells that contribute to cortical morphogenesis. b | Subplate (SP) neurons (step 1) and Cajal-Retzius (CR) cells (step 2) migrate at the onset of corticogenesis and settle under the cortical plate or the pia, respectively. Different subtypes of CR cells are generated during development (light blue and pale green). Projection neurons (PNs; light pink) are generated by radial glia (RG) cells and are first multipolar. They undergo a non-directed migration and become bipolar to engage in locomotion on RG cell fibres (step 3). They reach their final location by detaching from these fibres and undergoing a final somal translocation. The cortical interneurons (cINs) are born in the subpallium and migrate tangentially along defined streams (St1 and St2) that are running in the cortical wall before reaching their ad hoc cortical layer (step 4). Additional cell populations reach the cortex during development and include the microglia, which mostly use vessels to enter the forebrain (step 5). They disseminate into the forebrain parenchyma using amoeboid-like movements. Other populations that reach the cortex by tangential migration include the oligodendrocyte precursors (OPCs; lime green) that are born in the ventral forebrain (step 6). Corridor cells are located in the subpallium and migrate ventrally to allow the progression of thalamocortical (TC) fibres (step 7). Arrows represent the direction of migration of microglia (dark blue). E, embryonic day; TH, thalamus.

Leading process

Principal neurite of a migrating cell located ahead of the soma that contributes to its navigation along a migration path.

Polarity reversal Inversion of the polarity of a migrating cell along its moving axis. among GE progenitors in mice. However, a limited set of genes expressed differentially in cIN progenitors prime the cells for individual differentiation and maturation trajectories into different interneuron subtypes, which further consolidate during their migration towards the cerebral cortex ${ }^{6,7}$. cINs are mainly generated in the medial and caudal GEs (MGE and CGE, respectively) and are repelled from these regions by diffusible cues such as SLIT1, netrin 1 and ephrin A5 $\left(\mathrm{REFS}^{8-10}\right)$. They navigate tangentially along distinct migratory streams that are dynamically remodelled during development ${ }^{11}$. The choice of a migratory path correlates, to some extent, with the temporal origin and fate of $\mathrm{CINs}^{12}$ and is determined by permissive and chemoattractant cues, such as neuregulin 1 and CXCL12 (REFS ${ }^{13,14}$ ). The direction of migration also relies on the dynamic branching of the leading process ${ }^{15}$ and polarity reversal ${ }^{16}$. By the end of embryogenesis, cINs disperse in the cortical plate (CP) using their primary cilium to sense sonic hedgehog $(\mathrm{SHH})$ gradients $^{17,18}$ and undergo radial and/or oblique migration to reach their cortical layers ${ }^{19}$ and functionally connect to partners. The migration of cINs in the forebrain parenchyma is discontinuous and alternates advancements with pauses. At the molecular level, this processes is paced by cytoskeleton-generated forces where microtubules recruit organelles and actomyosin 


\section{Nucleokinesis}

Forward displacement of the

nucleus in the leading process

of a migrating neuron driven by

dynamic changes of the

cytoskeleton and in particular

actomyosin contraction taking

place around the nucleus.

In moving neurons, the

nucleokinesis is cyclic and

paces their migration.

Subpallial sorting

Biological process contributing

to the sorting of cells born in

the ventral forebrain (the

subpallium) into the dorsal

forebrain (the pallium) contractions drive nucleokinesis ${ }^{20}$. The pausing time of cINs represents around $40 \%$ of the migration process and requires a cyclic reduction in myosin light chain kinase (MLCK) activity via its processing by the cytosolic carboxypeptidase 1 (REF. ${ }^{5}$ ). Pauses during migration are not synchronized, thus generating heterogeneity of movement within the cIN population to regulate their subpallial sorting and cortical invasion. The regulated sorting of cINs shapes cortical morphogenesis via a crosstalk with dorsal cortical progenitors, the nature of which remains to be determined ${ }^{5}$.

As the migration of cINs into the cortex takes place in parallel with the production and migration of other cell types (FIG. 1 b), it would be interesting to decipher whether mechanisms generating heterogeneity of cell movement are also at play during the colonization of the cortex by other cell populations.

Multimodal migration of projection neurons. PN fate diversity is controlled by successive transcriptional waves in cortical progenitors ${ }^{21}$. At the onset of corticogenesis, PNs spread radially throughout the entire cortical wall and reach their final position by somal translocation. At mid-corticogenesis, the subsequent waves of newborn PNs have no basal attachment to the pia and undergo a complex migration process. They first become multipolar and move in random directions ${ }^{22}$ before repolarizing to initiate locomotion along RG fibres ${ }^{23}$. The multipolar-to-bipolar transition, the exact function of which remains elusive, is orchestrated by several transcription factors which, together with cytoplasmic and membrane effectors, promote the dynamic rearrangement of the cytoskeleton and centrosome positioning during migration ${ }^{24}$. PNs also undergo moderate nucleokinesis during locomotion, a process driven by actomyosin contraction, endocytosis and cell adhesion events ${ }^{25}$. PNs anchor their leading process in the marginal zone at the end of their locomotion to undergo a final somal translocation to settle at their final position ${ }^{16}$ (FIG. 1 b).

Pioneering migration of transient neurons during cerebral cortex development. Several neural cell populations that reach the cortex by tangential migration are eliminated during brain maturation in rodents. These include the transient glutamatergic neurons composed of the Cajal-Retzius (CR) cells, the subplate (SP) cells and the CP transient (CPT) neurons. SP cells and CPT neurons are born in the rostral medial telencephalic wall and ventral pallium/pallium-subpallium boundary (PSB), respectively ${ }^{26-28}$. Tangentially migrating SP neurons follow a trajectory parallel to the pia to reach the preplate (PP) and later settle under the $\mathrm{CP}^{26,29,30}$. SP neurons generate the first axons that project out of the cortex and are postsynaptic targets for afferent thalamocortical (TC) axons $\mathrm{s}^{31,32}$. Additionally, SP neurons guide corticofugal projections, shape intracortical connectivity and promote the formation of cortical networks in rodents and primates ${ }^{33-36}$. A fraction of non-tangentially migrating SP neurons are generated by RG cells and migrate along their radial processes ${ }^{26}$. Some reports suggest that SP neurons are still detected during the early postnatal periods both in rodents and primates ${ }^{37,38}$.
CPT neurons migrate tangentially into the cortex and exert non-cell-autonomous modulation of cortical neurogenesis by regulating the cell cycle length and proliferation rate of cortical progenitors ${ }^{28}$. The larger transient population is represented by the CR cells that colonize the brain before $\mathrm{CP}$ formation and disappear by the end of the first postnatal week in mice ${ }^{39}$. In rodents, CR cells are generated in three distinct regions of the forebrain - the hem, the PSB and the septum ${ }^{40}$ - and disperse homogeneously under the cortical surface via contact-repulsion migration ${ }^{41}$ (FIG. 1 b). Mathematical models show that the distribution of CR cells originating from different locations is regulated by contact repulsion and by several intrinsic properties, such as the onset, speed and directionality of migration ${ }^{42}$. However, in the human cortex, the classification of CR cells is more complex and includes the transient CR (tCR) and the permanent $\mathrm{CR}(\mathrm{pCR})$ cells ${ }^{43}$. pCR cells might share a function with the postnatal rodent CR cells by contributing to the organization of cortical layering and circuitry ${ }^{44}$. It is worth noting that the maintenance of these cells in the human cortex during adulthood is subject to controversy as they share morphological features with some GABAergic interneurons, and co-detection of their specific markers (for example, TBR1 and calretinin) is found only in the hippocampus ${ }^{45}$.

Glial cell migration in the developing cerebral cortex. Despite their number, neurons represent only a fraction of cells that compose the cortex and its functional units. Microglia, originating from yolk sac myeloid progenitors, form one large non-neuronal population that invades the forebrain at the onset of neurogenesis ${ }^{46}$. These cells contribute to developmental functions that go beyond their phagocytic activity ${ }^{47,48}$. Upon entrance in the dorsal forebrain, microglia begin to colonize from the pial surface, following dorsal-to-ventral and rostral-to-caudal gradients $^{49,50}$ (FIG. 1 b). $\mathrm{CX}_{3} \mathrm{CL} 1-\mathrm{CX}_{3} \mathrm{CR} 1$ signalling is required for their infiltration, proliferation and distribution in the developing brain. Microglia constitute a self-sustained population that proliferates during late gestation and postnatal periods. The exponential increase in microglia number after birth raises the possibility that blood monocytes or bone-marrow-derived cells might also reach the brain and add or substitute embryonically generated microglia ${ }^{46}$. At early stages of neurogenesis and during adulthood, microglia often associate with progenitors and dying cells ${ }^{50-52}$. Apoptotic cell clearance is a driving force for microglia invasion ${ }^{53}$. Colonization of the $\mathrm{CP}$ by microglia requires $\mathrm{RG}$ processes and blood vessels, takes place from E16 onwards and follows an inside-out gradient ${ }^{48,54}$. Once they reach the cortical wall, these cells are attracted to the ventricular zone (VZ) and subventricular zone (SVZ) by the CXCL12 released by intermediate progenitors (IPs), which activates CXCR4 or CXCR7 receptors in microglia ${ }^{55}$. Additional molecules have been reported to recruit or facilitate microglia migration within the forebrain (for example, colony-stimulating factor 1 (CSF1), interleukin-34 (IL-34), VEGFR1 acting in synergy with CSF1 in the SVZ, superoxide ions and metalloproteinases (MMP8 and MMP9)) (reviewed in REF. ${ }^{56}$ ). 


\section{Box 1 Cell migration in the cortex across evolution}

The laminar organization of the cortex along its dorsoventral axis and the expansion of its layers are novel acquisitions of evolution ${ }^{126}$, which required adaptation of the migration behaviour of resident cell populations. For example, somal translocation of early projection neurons (PNs) is mammalian-specific and has been introduced during evolution to efficiently convey positional information from the ventricular zone (VZ) to the corte ${ }^{127}$. Later during corticogenesis, sibling PNs move laterally before locomoting on radial glia fibres, thereby contributing to cortical expansion ${ }^{84,128}$. Despite originating from different progenitors, cortical interneurons (cINs) from sauropsids and mammals integrate the pallium via a conserved tangential migration ${ }^{129}$. Guidance cues are preserved, but transplantation of non-mammalian cINs into the mammalian cortex showed that they cannot enter the cortical plate ${ }^{130}$. Similar to rodents, primate cINs originate from ganglionic eminences (GEs) and undergo tangential migration ${ }^{131}$. Recent studies identified novel late-migrating cINs generated in the subventricular zone and targeting the frontal lobe of young children. Their delayed incorporation into the cerebral cortex may support the extension of plasticity periods in humans ${ }^{132}$. The refinement of some human cortical functions required the formation of novel circuits, and migration of neurons from the GEs to the dorsal thalamus may have synchronized the co-evolution of the frontal cortex and the thalamic nuclei ${ }^{13,134}$. The formation of thalamocortical (TC) connections relies on corridor cells that have remarkably conserved guidance properties across species. During evolution, a reorientation of corridor cells from the lateral GE to the non-permissive medial GE opened a novel internal path for thalamic axons ${ }^{135,136}$. This evolutionary step might have been essential for improving TC communication.

\section{Anterior entopeduncular} area

Region of the ventral forebrain that hosts progenitors of

GABAergic and cholinergic interneurons as well as oligodendrocyte progenitor cells.

Neocortical arealization Biological process that organizes the tangential subdivision of distinct neocortical fields responsible for computing higher cerebral functions.

\section{Morphogens}

Non-uniformly distributed

molecules that govern

biological processes

contributing to morphogenesis

in a developing organism

Multipolar migration Cell migration mode

characterized by the continuous extension and retraction of new neurites from the soma
Another important pool of glial cells that colonize the cerebral cortex concurrently with cINs are the oligodendrocyte precursor cells (OPCs), most of them becoming the myelinating cells of the $\mathrm{CNS}^{3}$. In the mouse, the first wave of OPCs is generated at E11.5 in the MGE and the anterior entopeduncular area. This wave of OPCs is followed by a second one, born in the lateral GE (LGE) and CGE at E15.5 (FIG. 1a,b), and a third one arising after birth within the cortical wall ${ }^{3}$. Experimental ablation showed that OPCs from different lineages can compete for forebrain territories ${ }^{3}$. The navigation of OPCs in the cortical parenchyma requires the decoding of gradients of attractive cues, such as PDGFRa ${ }^{57}$, and repulsive ones, such as the BMPs secreted by the meninges ${ }^{58}$. In addition, OPCs may interact with vessels via WNT-dependent signalling to colonize the pallium and later migrate from deep to more superficial layers in the cortex ${ }^{59}$. However, OPCs can migrate in vitro in biological preparations devoid of endothelial cells ${ }^{60}$, suggesting that vessels might be only one of the many substrates used by these cells to migrate. Interestingly, their association with blood vessels prevents their differentiation ${ }^{59}$. Despite accumulating studies focusing on OPC motility, the exact migration mode adopted by the OPCs from the different waves remains undescribed.

The cerebral cortex is thus composed of distinct neuronal and non-neuronal cell types, the study of the diversity of which is currently being revisited by single-cell biology $y^{6,761,62}$. These cells settle either transiently or permanently (depending on type) in the cortical wall after having used distinct migration strategies adapted during evolution to face the physical constraints appearing in larger and more complex brains (BOX 1).

\section{Cellular crosstalk promotes morphogenesis Cerebral cortex arealization}

The cortex is not homogeneous in size and function along the anteroposterior axis. Its tangential organization segments the cortical surface into primary and secondary motor, sensory (visual, somatosensory or auditory areas) and associative areas. Each field has a specific pattern of connection that generates cortical networks producing behaviours. In primates, higher-order functions correlate with the expansion of cortical areas responsible for integrative functions and higher-order processing such as Broca's area, composed of the somatosensory cortex, motor cortex and premotor cortex and controlling speech in humans ${ }^{63}$. Neocortical arealization is primarily the result of the action of morphogens secreted early by patterning centres in proliferative cell populations. Molecules such as BMP, FGF, SHH and WNT allow the establishment of the dorsoventral and anteroposterior axes and in turn generation of the different cortical fields ${ }^{64}$. In addition to these morphogenic gradients, postmitotic populations interplay to shape the tangential cytoarchitecture of the cortex. One of these populations is the CR cells, which have an important role for cortical patterning. CR cells born in distinct forebrain regions differ in their molecular identity ${ }^{40}$. Each subpopulation migrates from its birthplace to invade the cortical surface and colonize distinct domains of the cortex ${ }^{65}$. Interestingly, CR cells migrate in the vicinity of RG cell fibres and disseminate before the onset of cortical neurogenesis. Loss of CR cells impairs the regionalization of the neuroepithelium ${ }^{65}$; however, the molecular dialogue existing between CR and RG cells remains to be identified.

The modification of the migration speed of one CR neuron subpopulation leads to a change in the cortical surface covered by each subpopulation. In turn, this change leads to a proportional change in the size of higher-order cortical territories normally associated with a particular subtype of CR cells ${ }^{42}$.

Other populations of postmitotic cells can also influence cortical arealization. For example, patterning of the different areas of the sensory cortex results from the regulation and input from TC axons ${ }^{66-68}$. Removal or increase of some TC afferent fibres results in postnatal alteration of the size of the primary visual cortex ${ }^{69,70}$ but has little effect during development ${ }^{71}$. The exact nature of the signalling triggered by the TC fibres in the somatosensory cortex remains elusive, but interaction with postnatal PNs through cadherin has been suggested ${ }^{72}$.

\section{Cerebral cortex lamination}

The vertical lamination of the cortex depends on accurate spatial positioning and interaction between transient (RG cells, CR cells and some SP neurons) and permanent (cINs, PNs and glia) cell populations within the extracellular matrix (BOX 2). When RGs switch to a neurogenic mode of division (around E12.5 in mice), CR cells have already covered the entire cortical surface ${ }^{26}$. Together with the SP neurons positioned above the proliferative VZ, they form the PP, an instructive structure for migration and positioning of $\mathrm{PNs}^{73}$. Newborn PNs experience a slow multipolar migration until they reach SP neurons, with which they establish transient glutamatergic synapses that generate NMDA receptor-mediated signal transmission and $\mathrm{Ca}^{2+}$ entry ${ }^{73}$ (FIG. 2). $\mathrm{Ca}^{2+}$ transients are important for the morphological conversion of newborn PNs into fast bipolar locomoting PNs along 
Box 2 | The extracellular matrix is a key regulator of neural cell movements

All brain cells face complex and changing extracellular microenvironments. The extracellular matrix (ECM) constitutes the first line of interaction between neural stem cells, migrating cells and postmitotic neurons with their environment ${ }^{137}$. The ECM forms a basal lamina around the brain and its vasculature ${ }^{138,139}$ and has a fundamental role in the formation and function of the blood-brain-barrier and in the establishment of proper cortical lamination ${ }^{140,141}$. Impaired layering can result from disturbed interaction between radial glia (RG) cells and the cortical marginal zone (MZ) ${ }^{142}$. Moreover, disruption of $\beta 1$ integrin or other components of the basement membranes of the meninges leads to RG death, which correlates with a decreased brain size ${ }^{143}$. The ventricular zone also contains receptors for cell adhesion that are fundamental to anchor and stabilize RG apical processes ${ }^{144,145}$. Cell adhesion proteins are used by migrating neurons to form adhesive interactions with other neurons, glial cells and the ECM. These interactions are mainly mediated by integrin signalling with some specificity in different types of neuron. For example, interneurons use $\alpha 3 \beta 1$ integrin to sense and respond to netrin 1 , a ligand contributing to a permissive tangential path for migrating cortical interneurons ( $\mathrm{cINs}$ ) in the $\mathrm{MZ}^{146}$. Additional contributions from ECM molecules have been demonstrated, such as chondroitin-4-sulfate acting in concert with semaphorin $3 \mathrm{~A}$ to guide migrating $\mathrm{cINs}^{147}$. However, it remains unknown whether spatiotemporal changes in ECM composition actively contribute to control key cellular events that shape cerebral cortical morphogenesis.

RG fibres ${ }^{73}$. However, bipolar conversion of PNs occurs in reelin-signal-deficient mouse models ${ }^{74}$, where SP neurons are misplaced under the $\mathrm{pia}^{75}$, suggesting that this process might involve several mechanisms.

CR cells are located under the pia and release reelin, which controls the repolarization of migrating $\mathrm{PNs}^{76}$ and later instructs them to stop their migration ${ }^{76,77}$ (FIG. 2). Once released, reelin forms a positive gradient from the uppermost layer of the cortex (layer I), where CR cells reside. The release of reelin from CR cells allows newborn PNs to migrate beyond their older siblings and build an inside-out cortex. However, removal of the cortical hem, a source of CR cells, does not generate cortical layer abnormalities ${ }^{78}$, suggesting that the function of these cells might be replaced by other cell types or structures. Importantly, these cells play additional functions such as specifying the identity of the distal dendritic compartment of upper-layer neurons ${ }^{79}$ and controlling the timing of migration of cINs in the cortex ${ }^{80}$. They also integrate maturing neuronal networks, receiving excitatory and inhibitory inputs and signal to pyramidal neurons through long-range horizontal axonal projections ${ }^{81}$.

Together with SP neurons, CR cells shape cortical architecture in synchrony with RG cells to produce an appropriate number of PNs that populate the cortical layers and acquire the appropriate molecular identity. The vertical organization of the cortex is translated into a columnar organization of neurons that share an overlapping sensory receptive field. Neurons composing a minicolumn are densely interconnected and respond synchronously to electric stimulation ${ }^{82}$. The minicolumns are functional units and have been proposed to reflect the radial migration of clonally related PNs originated from a single RG cell ${ }^{83}$. However, minicolumns are not strictly clonally related, and the first phase of multipolar migration allows a certain degree of lateral spreading $^{84}$. This leads PNs of different origins to intermingle and allows the column to be populated by correct neuronal subtypes.

\section{Cellular crosstalk in corticogenesis}

The activity of the cerebral cortex relies on functional networks composed of excitatory PNs and inhibitory cINs, which are born from distinct pools of forebrain progenitors. The laminar distribution of cINs depends on cues provided by $\mathrm{PNs}^{85}$, and the neuronal excitatoryinhibitory (E/I) ratio is established during the maturation of the cortex and is later stabilized by synaptic mechanisms ${ }^{86}$. An altered proportion of PNs and cINs leading to a disruption of the E/I balance has been associated with epilepsy ${ }^{87}$ and psychiatric disorders (reviewed in $\mathrm{REF}^{88}$ ). Multiple cellular and molecular mechanisms are at play during the maturation of the cortex to control the bona fide proportion of PNs and cINs that are connecting to each other in the cortical wall. Indeed, the number of migrating cINs reaching the cortical wall during development exceeds the final number, but the $\mathrm{E} / \mathrm{I}$ balance is further adjusted via regulated apoptosis of supernumerary $\mathrm{cINs}^{89}$. Their elimination relies on the early establishment of local cortical circuits where PNs regulate cIN survival through a synaptic-dependent mechanism ${ }^{90,91}$. Recent studies have highlighted additional molecular crosstalk between cortical progenitors and migrating neurons that contribute to the establishment of the cortical cytoarchitectonic. The developing cortical wall is composed of IPs that serve as transient amplifying progenitors to generate most superficial PNs. In addition, IPs promote the migration of cINs in the path between the intermediate zone and the SVZ by releasing diffusible signals, among which the chemokine CXCL12 signals to CXCR4 receptors expressed at the surface of $\mathrm{CINs}^{14,92}$ (FIG. 2). The controlled migration of cINs in streams canalizes their navigation and may concentrate morphogenic signals released by them. Indeed, while migrating in the vicinity of IPs, cINs release diffusible cues that modulate IP proliferation, thereby affecting the production of superficial $\mathrm{PNs}^{5}$ (FIG. 2). Thus, IPs and migrating cINs establish important bidirectional crosstalk to control their position and numbers during the formation of the cerebral cortex. It is worth noting that migrating cINs also control the biology of OPCs via the release of fractalkine, which stimulates their differentiation without affecting their proliferation ${ }^{93}$.

Early migrating PNs control cortical progenitor fate via multiple non-cell-autonomous mechanisms. For example, SIP1 expression in early-born PNs prevents premature expression and release of NT3 and FGF9 that signal back to cortical progenitors to promote advancement of the sequential production of upper-layer PNs, deep-layer PNs and glia ${ }^{94}$ (FIG. 2). Another important signalling initiated by newborn PNs goes through their ability to sense WNT7 and express Jagged 1 (JAG1) as a result of the activation of a non-canonical WNT signalling. According to their proximity with RG cells, PNs further mediate a JAG1-activation of Notch to control the sequential production of PNs and glia ${ }^{95}$ (FIG. 2).

\section{Migrating glia shape cortical circuits}

During development, microglia control cortical progenitor numbers via phagocytosis. Moreover, they eliminate some progenitors after the neurogenic period ${ }^{51}$, suggesting that microglia also regulate gliogenesis in the 
a

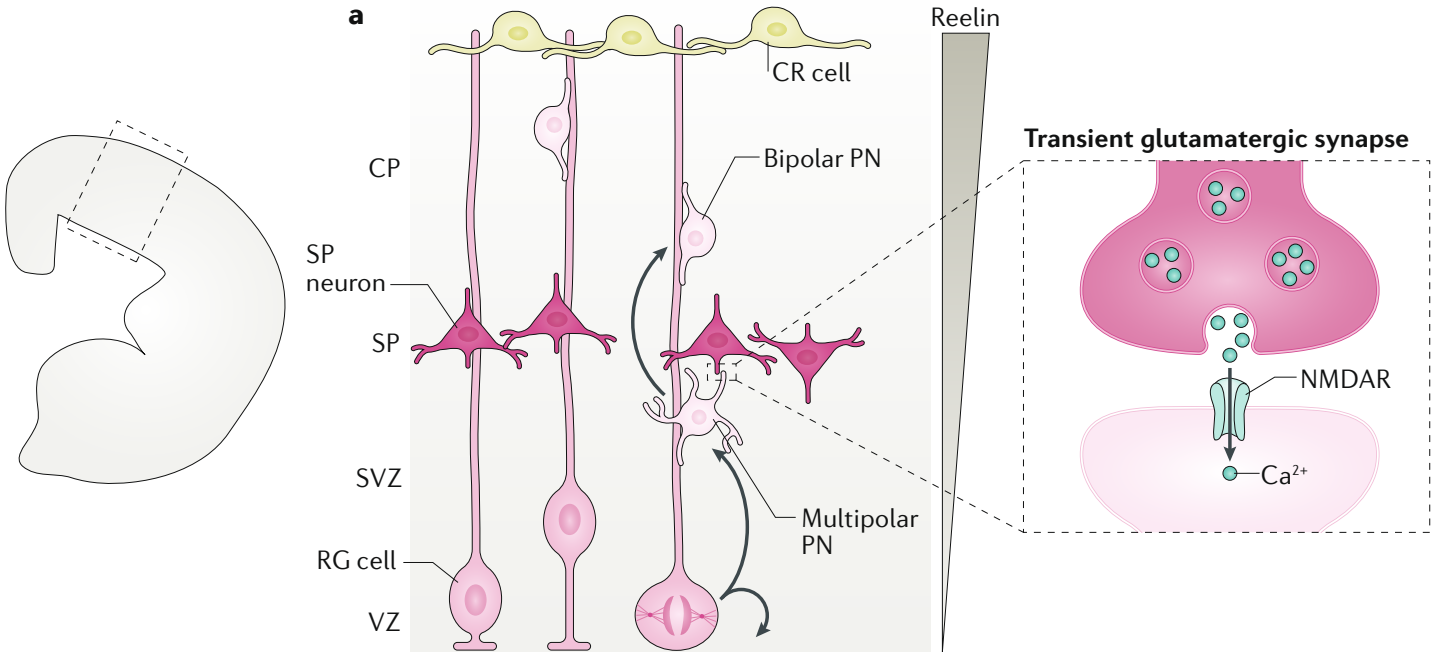

b

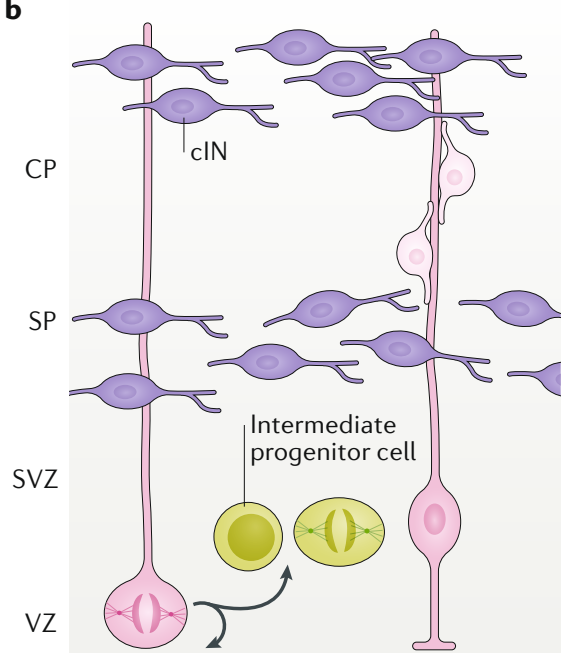

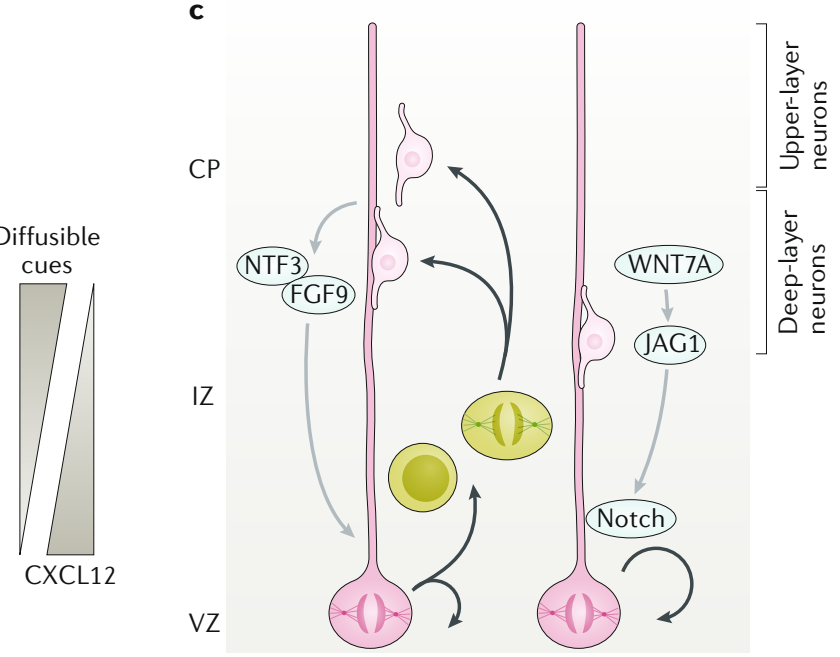

Fig. 2 | Cellular crosstalk shapes cerebral cortical morphogenesis. a | At the onset of corticogenesis, projection neurons (PNs) that are born in the ventricular zone (VZ) by asymmetric division of radial glia (RG) cells are multipolar. They migrate through the subventricular zone (SVZ) towards subplate (SP) neurons and establish transient glutamatergic synapses with these neurons. NMDA receptor (NMDAR)-induced $\mathrm{Ca}^{2+}$ entry contributes to the morphological conversion into bipolar cells, which then migrate on RG cell fibres through the cortical plate (CP). Cajal-Retzius (CR) cells are located at the top of the $\mathrm{CP}$ and release reelin, which establishes a gradient (grey triangle) contributing to the repolarization of PNs and the arrest of their migration. $\mathbf{b}$ | Migrating cortical interneurons (cINs, purple) and intermediate progenitors (IPs, lime green) in the cortical wall engage in bidirectional crosstalk (including diffusible cues such as secreted CXCL12), which controls the recruitment of cINs and the proliferation of IPs. By regulating IP proliferation, cINs regulate the final numbers of upper-layer PNs (light pink). c| Newborn PNs expressing the transcription factor SIP1 (light pink cells) control the fate of the RG daughter cells via the release of NT3 and FGF9 as well as the activation of a Notch-dependent signalling pathway in RG cells. These mechanisms control the sequential generation of PNs for deep and upper layers as well as glia arising later from dividing cortical progenitors. IZ, intermediate zone.

cortex. Microglia have functions beyond phagocytosis by trimming non-myelinated axons, contacting synaptic elements and regulating spine density in postnatal periods and engulfing and/or promoting synaptic contact formation (reviewed in $\mathrm{REF}^{4}$ ). While migrating, microglia might also contribute to blood vessel formation as suggested by their close association with endothelial cells $^{96-98}$, and microglia control cortical invasion of $\mathrm{cINs}^{48}$ and influence OPC maturation ${ }^{99}$. Strikingly, not much is known about the migration properties of OPCs and in particular about those generated in the subpallium ${ }^{3}$. In the early postnatal period, some OPCs can establish synaptic contacts with neurons and receive GABAergic inputs that are important to control their population size ${ }^{100,101}$. However, it remains uncertain whether these early networks established between PNs or cINs and OPCs involve the ventrally generated OPCs.

These examples support the existence of abundant dynamic crosstalk among neural cells invading and settling into the cortex to support its morphogenesis. However, the complexity of such crosstalk in vivo limits a full understanding, and in silico modelling has recently been introduced to shed new light on the regulatory mechanisms at play. 




Fig. 3 | Innovative tools to study neuronal migration of human-derived neurons. Assembloids are fused organoids patterned towards subpallial and pallial fates. On dissociation and culture in a microfluidic device, they can be used to follow neuron migration from its subpallial to pallial entity and thus untangle cell-autonomous from non-cell-autonomous components. Time-lapse analysis of assembloids might enable assessment of the impact of interneuron migration on proliferation of ventricular zone (VZ)-like and subventricular zone (SVZ)-like progenitors and their cell cycle exit. This analysis can be supported by in silico modelling. Cell diversity and maturation in assembloids could be assessed by coupling single-cell tracing with single-cell transcriptomics and the progressive establishment of its cytoarchitecture followed by immunostaining upon tissue clearing. cIN, cortical interneuron; PC. progenitor cell.

\section{Understanding cortical cytoarchitectonics In vitro modelling}

Many different in vitro assays have been used to characterize neural migration behaviour and its biochemical and molecular regulation (FIG. 3). Such assays include live imaging of brain organotypic slices or explants to follow neuronal migration ${ }^{102}$, the Boyden chamber assay and microfluidic devices to study the migration of distinct cell types under the chemoattraction of selective molecules $^{5,18}$. Interestingly, as novel biomaterials develop, these assays are increasingly being revisited as new tools for the study of neuronal migration. Bioscaffolds mimicking the extracellular matrix can be engineered into compartmented space and nanotopography in culture by using synthetic materials such as polyethylene glycol (PEG) or hydrogels. Moreover, photoactive materials are being used to print proteins and generate local mechanical forces on the substrate or guide local cell seeding ${ }^{103}$. PEG hydrogel membranes can also be photopatterned in situ to generate microfluidic environments, constituting a flexible tool to study cell migration ${ }^{104}$.

Understanding how human brain cytoarchitecture is established is a major challenge of developmental biology. The limiting factors are the complex organization of the human brain and the poor accessibility to human tissue. Emerging culture systems are being made available to generate simple to complex human self-organized 3D structures named organoids, where understanding of cell population interactions becomes possible in vitro. Organoids are generated by culturing human pluripotent stem cells in suspension, which aggregate and self-organize to form 3D structures with features of developing brains in vivo ${ }^{105}$. Together with technical advances such as CRISPR-Cas9 to produce isogenic cells lines by genome editing, tissue clarification techniques and light sheet microscopy for large-scale 3D imaging, organoids have become a critical tool to study human cell population interactions and tissue morphogenesis. The 'assembloid' is a recent technological advancement of organoids to study human neuronal migration ${ }^{106,107}$. It consists of a fusion of brain organoids patterned with morphogen addition in their culture media to generate different brain regions of interest or brain regions that cannot be properly specified together ${ }^{106,108}$. Human brain assembloids could help to decipher whether migrating cINs control the proliferation of IPs as shown in rodents ${ }^{5}$ and how cIN subtype specification ${ }^{6,7}$ and survival ${ }^{91}$ are controlled. Future improvements should allow the functional assembly of a wide range of distinct brain regions to understand how they influence each other to generate cortical cytoarchitecture and neuronal circuits.

\section{In silico modelling}

Modelling helps in understanding the developmental processes in many different ways. Modelling can be used at different scales - from isolated mechanisms that have 
Random walk

Cell migration mode

characterized by constant

changes of movement angle. an impact at the single-cell level to models allowing the representation of an entire cell population or organs. Models predict phenotypic outcomes upon modification of input parameters and give rise to new testable hypotheses. Models have some limitations: small-scale models simplify complex processes in a limited developmental window to test specific hypotheses isolated from larger-scale and more complex phenotypes. On the other hand, multiscale and integrative models render developmental processes from molecular signalling to tissue and organ formation possible, but they require a large amount of data and extensive acquired knowledge.

Comprehensive modelling has been used to understand the establishment of cortical cytoarchitecture, neuronal migration and cell population interactions. Cortical development has been simplified in silico to represent neurogenesis and the establishment of its radial organization. To understand PN production, experimental parameters such as duration of the cell cycle, rate of cell cycle exit and cell survival have been used to derive mathematical models that can predict neuronal output across species $^{109,110}$. Later, these models were refined by integrating novel data acquired from the developing (for example, number of progenitor cells) and mature brain (for example, number and distribution of PNs) of different species ${ }^{111}$. The upgraded model predicts not only neuronal output, layer formation and cell progenitor dynamics across species but also the spatiotemporal particularities of cortical formation, such as increased upper-layer occupancy with increased neuronal output and spatial neuronal density variation across the primate cortex. Mathematical models accounting for cell proliferation and neuronal production have shown how the perturbation of proliferation regulators and timing of neurogenesis affect neuronal output, cortical thickness and surface size (for example, upon LHX2 mutation $)^{112}$. Building on these studies by adding adequate

\section{Box 3 | Studying migration properties at the single-cell level}

The understanding of the mechanisms governing cerebral cortical development has recently benefited from the blooming of single-cell biology. While accumulating data are being generated by single-cell transcriptomics to uncover cell diversity in the cerebral cortex ${ }^{6,7,61,62}$, clonal analysis has been used to label isolated progenitors and monitor their progeny. Clonal tracing was originally performed by infecting tissues with a low titre of retroviruses and lentivirus or by expressing plasmids coding for a fluorescent reporter ${ }^{148,149}$. Technological developments were recently made, including the use of endogenous retroelements such as long interspersed nuclear element 1 (LINE1) to trace cell lineage in humans ${ }^{150}$ as well as a transposon-based technique such as the CLoNe method to target single neural progenitors in mice and chickens ${ }^{151}$. Genetic animal models include the Brainbow mouse, in which clonally related cells are labelled in a wide range of colours depending on the random expression of different fluorophores upon Cre recombination ${ }^{152}$. MAGIC (multi-addressable genome-integrative colour) is a genome-integrative transposon-based strategy derived from the Brainbow concept allowing labelling of different subcellular compartments ${ }^{153}$. Another genetic approach named mosaic analysis with double markers (MADM) has recently been developed to more precisely map the lineage of mouse cortical progenitors and the migration of their progenies ${ }^{154}$. This genetic toolbox can also be engineered at the locus of the gene of interest and coupled with knockout strategies to distinguish cell-autonomous and non-cell-autonomous regulation ${ }^{155}$. These genetic tools advance our knowledge and shed some new light on cell migration processes in the brain. For example, it has been suggested that clonally related cortical interneurons are located in close proximity ${ }^{156,157}$, but a combination of MADM with barcode viral infections showed that these cells disperse over long distances in the cortex ${ }^{158}$. progenitor cell types, cortical development models have also demonstrated the strong adaptability of cortical progenitors to cope with postmitotic neuronal cell death during corticogenesis by reducing IP cell cycle length and by promoting self-renewing divisions ${ }^{113}$. Other models have been established to predict how neuronal migration is regulated in the cortex. The radial migration of PNs was also modelled using knowledge of a simplified network of known migration regulators: GABA, reelin, doublecortin (DCX) and LIS1. This model recapitulated the different phases of PN migration from the apical surface and their multipolar-to-bipolar transition and locomotion across neuronal layers. The model also predicted migration defects upon LIS1 and DCX loss of function ${ }^{114}$. On a larger scale, mathematical models can envision how cells migrate and how their movement influences other cell populations and cortical development. Studying the dissemination of CR cells under the cortical surface is a good example of a successful application of this type of approach ${ }^{41,42}$. As the repartition of CR cell subtypes during cortical development influences the identity of the cortical domain they occupy, deciphering the parameters regulating their migration and final repartition is essential to understanding how cortical cytoarchitecture is generated. Several migration parameters of CR cells were measured, and random walk and contact repulsion were also differently weighted to mimic the average displacement speed of each CR cell subtype. These data were integrated into a model able to predict how the average speed and directionality of migration differentially influenced the final distribution of each CR subtype at the surface of the cortex. Another example is the modelling of cortical invasion by cINs during development. Parameters of cIN migration, such as pausing and nucleokinesis, influence the number of cINs entering the developing mouse cortex. Shifting the stereotypical biphasic cIN migration mode of division towards a continuous displacement was predicted to lead to a higher homogeneity of cell movement and in turn to a larger recruitment of cINs invading the cortex ${ }^{5}$.

In silico models are evolving and being built upon with the addition of new data and recently acquired knowledge. For instance, models will improve by integrating interactomes and proteomic and transcriptomic screens at the population and single-cell level (BOX 3). The ultimate goal of mathematical modelling in this field is to represent cell population dynamics in sufficient detail so as to correctly predict the outcome of diverse perturbations occurring during cortical development. Once validated experimentally, such a model system will likely be relevant to understanding pathologies and to the design of therapeutic strategies.

\section{Cell migration in psychiatric disorders}

Each year, about 165 million people in Europe are affected by mental illness, representing a major cause of morbidity and impaired quality of life in affected individuals. The current view is that a large proportion of these disorders take root during brain formation via deviation from normal developmental trajectories. Although most of these neurodevelopmental disorders have a strong genetic basis, the precise aetiology of the 
condition often remains unknown. Thus, a major health challenge is to better understand their pathophysiological mechanisms to improve diagnosis and develop new pharmacotherapies that may ultimately lead to preclinical trials. Some genes linked to common neurodevelopmental disorders, such as autism spectrum disorder (ASD) and schizophrenia, regulate cortical neuron development, integration and their functional assembly in cortical circuits. For instance, recessive loss-of-function mutations in CNTNAP2 lead to a rare but highly penetrant form of ASD in humans characterized by a migration deficit of $\mathrm{PNs}^{115}$. Analyses conducted in Cntnap2-knockout mice ${ }^{116}$ not only support this observation but also reveal a reduction in cIN number ${ }^{117,118}$. FMR1 encodes FMRP, which is an mRNA-binding protein involved in translational regulation. FMR1 mutation leads to fragile X syndrome, which accounts for $2-3 \%$ of autism cases ${ }^{119}$. Similarly to the loss of function of CNTNAP2, FMRP deficit results in reduced migration of PNs into the $\mathrm{CP}^{120}$ and is also characterized by a lack of $\mathrm{CINs}^{121}$. Although more work is needed to understand the precise developmental sequence of pathological events leading to ASD, experimental analyses conducted with these animal models suggest that an early disruption of neuronal migration leads to abnormal cortical circuit assembly and function. Interestingly, research in animal models has shown that reversal of genetically based cortical malformation arising from neuronal migration defects is possible at early postnatal stages by reactivating specific molecular pathways. This finding is exemplified by the partial rescue of subcortical band heterotopia via re-expression of DCX in a rat model of $\mathrm{PN}$ migration defect ${ }^{122}$. As the first ASD symptoms are identifiable in the early months of childhood when the brain is plastic, this suggests that re-engineering the early postnatal cortex might be possible.

Recent technological developments of human organoid cultures ${ }^{105}$ open the path to foster our understanding of the pathophysiological mechanisms underlying ASD by using human-derived models. The recent generation of brain assembloids ${ }^{106,108}$ uncovered a novel migration deficit of cINs in Timothy syndrome, a monogenic disease associated with ASD and epilepsy ${ }^{106}$. The field of human organoids is growing at high pace and benefits from regular cell culture improvements ${ }^{123}$ and technological developments. As the in vitro modelling is getting closer to human brain development, human organoid cultures hold promise for untangling novel cellular crosstalk and movements underlying cortical morphogenesis in health and disease. Future technical improvements are required to increase the cellular diversity of organoids and their controlled growth over longer periods, allowing further neuronal differentiation and maturation of functional neural circuits. Long-term culture of organoids is currently limited by the poor diffusion of oxygen, nutrients and waste. Thus, improved methods such as $3 \mathrm{D}$ culture using microcarriers could prolong culture to increase the differentiation potential and increase cell-type diversity. Microencapsulation is another methodological approach to improve the oxygenation and delivery of nutrients and growth factors to the culture ${ }^{124}$.

\section{Conclusion and perspectives}

The complexity of the cerebral cortex cytoarchitectonic has long been fascinating biologists. Despite accumulating knowledge obtained with complementary in vitro and in vivo models, we do not fully understand the spatial and temporal coordination of mechanisms driving cerebral cortical morphogenesis. Recent progress suggests that migration not only is required for allocating final cell positioning in the cortex but, in some instances, also generates specific crosstalk for instructing local morphogenetic events via physical contact and/or release of diffusible cues. These processes underlie not only early corticogenesis steps, such as the rate of progenitor proliferation, but also the regulation of long-range communication between distant brain structures, initiation and refinement of synaptic communication and organization of higher-order areas responsible for cognition and complex behaviours.

By the time neurons wire together, some cortical cells have been eliminated in a regulated fashion. Although these cells actively contribute to morphogenetic crosstalk during development, the reason for their short life in the cortex after birth remains unclear but is often found to be conserved across evolution. This conservation suggests that their programmed elimination is required for the final maturation steps of the cortex and should be considered when generating large-scale modelling of neocortical microcircuitry ${ }^{125}$.

Despite considerable progress made during the past decade, we still do not have a comprehensive view on how cell migration contributes to morphogenesis, ultimately leading to the establishment of the complex cortical cytoarchitecture. Recent technological developments, such as single-cell biology, simplified 3D culture and in silico modelling for brain development, should soon improve the resolution of analyses to answer the remaining questions and likely raise novel ones. For instance, the nature of mechanisms of cell diversification in the brain and the exact contribution of intrinsic and environmental factors to this process remain unclear.

Recent single-cell analyses have revealed the existence of molecular signatures for newborn cINs that are otherwise difficult to identify because of their numerous subtypes, at times owing to the small size of their population or the late expression of landmark markers. This discovery should help monitor the migration of differentiating cINs in more detail and thus help to clarify whether the migration pattern of cINs as a whole population is homogeneous or whether migration patterns are specific to cIN subtypes. Moreover, neurons and glia undergo stereotypic migration patterns to reach their final position in the cortex, and it remains unknown whether such behaviour is intrinsically regulated or mainly dependent on environmental cues and/or cellcell interaction dynamics. The implementation of novel in silico models and predictive analyses will help us in deciphering to what extent neuronal migration is autonomously and/or non-autonomously driven at different developmental milestones and in distinct areas of the developing cerebral cortex.

Published online: 15 March 2019 
1. Marin, O. \& Rubenstein, J. L. Cell migration in the forebrain. Annu. Rev. Neurosci. 26, 441-483 (2003).

2. Heng, J. I., Chariot, A. \& Nguyen, L. Molecular layers underlying cytoskeletal remodelling during cortical development. Trends Neurosci. 33, 38-47 (2010).

3. Kessaris, N. et al. Competing waves of oligodendrocytes in the forebrain and postnatal elimination of an embryonic lineage. Nat. Neurosci. 9 173-179 (2006)

4. Thion, M. S. \& Garel, S. On place and time: microglia in embryonic and perinatal brain development. Curr. Opin. Neurobiol. 47, 121-130 (2017).

5. Silva, C. G. et al. Cell-intrinsic control of interneuron migration drives cortical morphogenesis. Cell 172 1063-1078 (2018)

\section{This work demonstrates the existence of a} crosstalk between migrating cINs and PN progenitors to control the output of upper-layer neurons.

6. Mayer, C. et al. Developmental diversification of cortical inhibitory interneurons. Nature $\mathbf{5 5 5}$, 457-462 (2018)

7. $\mathrm{Mi}$, D. et al. Early emergence of cortical interneuron diversity in the mouse embryo. Science $360,81-85$ (2018).

8. Hamasaki, T., Goto, S., Nishikawa, S. \& Ushio, Y. A role of netrin-1 in the formation of the subcortical structure striatum: repulsive action on the migration of late-born striatal neurons. J. Neurosci. 21. 4272-4280 (2001)

9. Marin, $O$ et al. Directional guidance of interneuron migration to the cerebral cortex relies on subcortical Slit 1/2-independent repulsion and cortical attraction. Development 130, 1889-1901 (2003).

10. Zimmer, G. et al. Ephrin-A5 acts as a repulsive cue for migrating cortical interneurons. Eur. J. Neurosci. 28 62-73 (2008)

11. Metin, C., Baudoin, J. P., Rakic, S. \& Parnavelas, J. G. Cell and molecular mechanisms involved in the migration of cortical interneurons. Eur. J. Neurosci. 23, 894-900 (2006).

12. Lim, L. et al. Optimization of interneuron function by direct coupling of cell migration and axonal targeting. Nat. Neurosci. 21, 920-931 (2018).

13. Flames, N. et al. Short- and long-range attraction of cortical GABAergic interneurons by neuregulin-1. Neuron 44, 251-261 (2004)

14. Tiveron, M. C. et al. Molecular interaction between projection neuron precursors and invading interneurons via stromal-derived factor 1 (CXCL12)/ CXCR4 signaling in the cortical subventricular zone/ intermediate zone. J. Neurosci. 26, 13273-13278 (2006).

15. Martini, F. J. et al. Biased selection of leading process branches mediates chemotaxis during tangential neuronal migration. Development 136, 41-50 (2009).

16. Nadarajah, B., Brunstrom, J. E., Grutzendler, J., Wong, R. O. \& Pearlman, A. L. Two modes of radial migration in early development of the cerebral cortex. Nat. Neurosci. 4, 143-150 (2001).

17. Baudoin, J. P. et al. Tangentially migrating neurons assemble a primary cilium that promotes their reorientation to the cortical plate. Neuron 76 1108-1122 (2012)

18. Higginbotham, H. et al. Arl13b in primary cilia regulates the migration and placement of interneurons in the developing cerebral cortex. Dev. Cell 23, 925-938 (2012)

19. Tanaka, D. H., Maekawa, K., Yanagawa, Y., Obata, K. \& Murakami, F. Multidirectional and multizonal tangential migration of GABAergic interneurons in the developing cerebral cortex. Development 133 , 2167-2176 (2006)

20. Bellion, A., Baudoin, J. P., Alvarez, C., Bornens, M. \& Metin, C. Nucleokinesis in tangentially migrating neurons comprises two alternating phases: forward migration of the Golgi/centrosome associated with centrosome splitting and myosin contraction at the rear. J. Neurosci. 25, 5691-5699 (2005)

21. Telley, L. et al. Sequential transcriptional waves direct the differentiation of newborn neurons in the mouse neocortex. Science 351, 1443-1446 (2016)

22. Tabata, H. \& Nakajima, K. Multipolar migration: the third mode of radial neuronal migration in the developing cerebral cortex. J. Neurosci. 23, 9996-10001 (2003).

23. Noctor, S. C., Martinez-Cerdeno, V., Ivic, L. \& Kriegstein, A. R. Cortical neurons arise in symmetric and asymmetric division zones and migrate through specific phases. Nat. Neurosci. 7, 136-144 (2004).
24. LoTurco, J. J. \& Bai, J. The multipolar stage and disruptions in neuronal migration. Trends Neurosci. 29, 407-413 (2006)

25. Kawauchi, T. et al. Rab GTPases-dependent endocytic pathways regulate neuronal migration and maturation through $\mathrm{N}$-cadherin trafficking. Neuron $67,588-602$ (2010).

26. Barber, M. \& Pierani, A. Tangential migration of glutamatergic neurons and cortical patterning during development: lessons from Cajal-Retzius cells. Dev. Neurobiol. 76, 847-881 (2016).

27. Pedraza, M., Hoerder-Suabedissen, A Albert-Maestro, M. A., Molnar, Z. \& De Carlos, J. A. Extracortical origin of some murine subplate cell populations. Proc. Natl Acad. Sci. USA 111, 8613-8618 (2014).

28. Teissier, A. et al. A novel transient glutamatergic population migrating from the pallial-subpallial boundary contributes to neocortical development J. Neurosci. 30, 10563-10574 (2010).

29. Bayer, S. A. \& Altman, J. Development of layer I and the subplate in the rat neocortex. Exp. Neurol. 107 48-62 (1990)

30. Hoerder-Suabedissen, A. \& Molnar, Z. Molecular diversity of early-born subplate neurons. Cereb. Cortex 23, 1473-1483 (2013)

31. Allendoerfer, K. L. \& Shatz, C. J. The subplate, a transient neocortical structure: its role in the development of connections between thalamus and cortex. Annu. Rev. Neurosci. 17, 185-218 (1994).

32. Molnar, Z., Adams, R. \& Blakemore, C. Mechanisms underlying the early establishment of thalamocortical connections in the rat. J. Neurosci. 18, 5723-5745 (1998).

33. Kanold, P. O., Kara, P., Reid, R. C. \& Shatz, C. J. Role of subplate neurons in functional maturation of visual cortical columns. Science 301, 521-525 (2003).

34. McConnell, S. K., Ghosh, A. \& Shatz, C. J. Subplate pioneers and the formation of descending connections from cerebral cortex. J. Neurosci. 14, 1892-1907 (1994).

35. Zhao, C. Kao, J. P \& Kanold, P O. Functional excitatory microcircuits in neonatal cortex connect thalamus and layer 4. J. Neurosci. 29 15479-15488 (2009)

36. Meinecke, D. L. \& Rakic, P. Expression of GABA and GABAA receptors by neurons of the subplate zone in developing primate occipital cortex: evidence for transient local circuits. J. Comp. Neurol. 317, 91-101 (1992).

37. Kostovic, I. \& Rakic, P. Developmental history of the transient subplate zone in the visual and somatosensory cortex of the macaque monkey and human brain. J. Comp. Neurol. 297, 441-470 (1990).

38. Woo, T. U., Beale, J. M. \& Finlay, B. L. Dual fate of subplate neurons in a rodent. Cereb. Cortex 1 , 433-443 (1991)

39. Hevner, R. F., Neogi, T., Englund, C., Daza, R. A. \& Fink, A. Cajal-Retzius cells in the mouse: transcription factors, neurotransmitters, and birthdays suggest a pallial origin. Brain Res. Dev. Brain Res. 141, 39-53 (2003).

40. Bielle, F. et al. Multiple origins of Cajal-Retzius cells at the borders of the developing pallium. Nat. Neurosci. 8, 1002-1012 (2005).

41. Villar-Cervino, V. et al. Contact repulsion controls the dispersion and final distribution of Cajal-Retzius cells. Neuron 77, 457-471 (2013).

42. Barber, M. et al. Migration speed of Cajal-Retzius Cells modulated by vesicular trafficking controls the size of higher-order cortical areas. Curr. Biol. 25 2466-2478 (2015).

This study shows that vesicular trafficking controls the cortical distribution of distinct CR cells, which influences the size and wiring of different cortical areas.

43. Meyer, G. \& Gonzalez-Gomez, M. The heterogeneity of human Cajal-Retzius neurons. Semin. Cell Dev. Biol. 76, 101-111 (2018).

44. Meyer, G. \& Gonzalez-Gomez, M. The subpial granular layer and transient versus persisting Cajal-Retzius neurons of the fetal human cortex. Cereb. Cortex 28 2043-2058 (2018).

45. Abraham, H. \& Meyer, G. Reelin-expressing neurons in the postnatal and adult human hippocampal formation. Hippocampus 13, 715-727 (2003)

46. Ginhoux, F. \& Garel, S. The mysterious origins of microglia. Nat. Neurosci. 21, 897-899 (2018).

47. Schafer, D. P. et al. Microglia sculpt postnatal neural circuits in an activity and complement-dependent manner. Neuron 74, 691-705 (2012).
48. Squarzoni, P. et al. Microglia modulate wiring of the embryonic forebrain. Cell Rep. 8, 1271-1279 (2014). This work suggests that the embryonic invasion of the brain by microglia controls the wiring of some forebrain circuits by affecting axonal growth and interneuron positioning.

49. Arnold, T. \& Betsholtz, C. The importance of microglia in the development of the vasculature in the central nervous system. Vasc. Cell 5, 4 (2013).

50. Ashwell, K. The distribution of microglia and cell death in the fetal rat forebrain. Brain Res. Dev. Brain Res. 58, 1-12 (1991).

51. Cunningham, C. L., Martinez-Cerdeno, $V$. $\&$ Noctor, S. C. Microglia regulate the number of neural precursor cells in the developing cerebral cortex. J. Neurosci. 33, 4216-4233 (2013).

52. Sierra, A. et al. Microglia shape adult hippocampal neurogenesis through apoptosis-coupled phagocytosis. Cell Stem Cell 7, 483-495 (2010).

53. Casano, A. M., Albert, M. \& Peri, F. Developmental apoptosis mediates entry and positioning of microglia in the zebrafish brain. Cell Rep. 16, 897-906 (2016).

54. Swinnen, N. et al. Complex invasion pattern of the cerebral cortex bymicroglial cells during development of the mouse embryo. Glia 61, 150-163 (2013).

55. Arno, B. et al. Neural progenitor cells orchestrate microglia migration and positioning into the developing cortex. Nat. Commun. 5, 5611 (2014).

56. Xavier, A. L., Menezes, J. R., Goldman, S. A. \& Nedergaard, M. Fine-tuning the central nervous system: microglial modelling of cells and synapses. Phil. Trans. R. Soc. B 369, 20130593 (2014).

57. Frost, E. E., Zhou, Z., Krasnesky, K. \& Armstrong, R. C. Initiation of oligodendrocyte progenitor cell migration by a PDGF-A activated extracellular regulated kinase (ERK) signaling pathway. Neurochem. Res. 34 , 169-181 (2009).

58. Choe, Y., Huynh, T. \& Pleasure, S. J. Migration of oligodendrocyte progenitor cells is controlled by transforming growth factor beta family proteins during corticogenesis. J. Neurosci. 34, 14973-14983 (2014).

59. Tsai, H. H. et al. Oligodendrocyte precursors migrate along vasculature in the developing nervous system. Science 351, 379-384 (2016).

60. Paez, P. M. et al. Golli myelin basic proteins regulate oligodendroglial progenitor cell migration through voltage-gated $\mathrm{Ca}^{2+}$ influx. J. Neurosci. 29 6663-6676 (2009)

61. Marques, S. et al. Transcriptional convergence of oligodendrocyte lineage progenitors during development. Dev. Cell 46, 504-517 (2018).

62. Pollen, A. A. et al. Molecular identity of human outer radial glia during cortical development. Cell 163 , 55-67 (2015)

63. Krubitzer, L. The magnificent compromise: cortical field evolution in mammals. Neuron 56, 201-208 (2007).

64. Hasenpusch-Theil, K., Watson, J. A. \& Theil, T. Direct interactions between $\mathrm{Gli} 3$, Wnt8b, and Fgfs underlie patterning of the dorsal telencephalon. Cereb. Cortex 27, 1137-1148 (2017)

65. Griveau, A. et al. A novel role for Dbx1-derived Cajal-Retzius cells in early regionalization of the cerebral cortical neuroepithelium. PLOS Biol. 8, e1000440 (2010)

66. Dehay, C., Horsburgh, G., Berland, M., Killackey, H. \& Kennedy, $\mathrm{H}$. Maturation and connectivity of the visual cortex in monkey is altered by prenatal removal of retinal input. Nature 337, 265-267 (1989).

67. Polleux, F., Dehay, C., Goffinet, A. \& Kennedy, H. Pre- and post-mitotic events contribute to the progressive acquisition of area-specific connectional fate in the neocortex. Cereb. Cortex 11, 1027-1039 (2001).

68. Borello, U., Kennedy, H. \& Dehay, C. The logistics of afferent cortical specification in mice and men Semin. Cell Dev Biol 76, 112-119 (2017).

69. Vue, T. Y. et al. Thalamic control of neocortical area formation in mice. J. Neurosci. 33, 8442-8453 (2013).

70. Chou, S. J. et al. Geniculocortical input drives genetic distinctions between primary and higher-order visual areas. Science 340, 1239-1242 (2013).

71. Garel, S., Yun, K., Grosschedl, R. \& Rubenstein, J. L. The early topography of thalamocortical projections is shifted in Ebf1 and Dlx1/2 mutant mice. Development 129, 5621-5634 (2002).

72. Egusa, S. F. et al. Classic cadherin expressions balance postnatal neuronal positioning and dendrite dynamics to elaborate the specific cytoarchitecture of the mouse cortical area. Neurosci. Res. 105, 49-64 (2016). 
73. Ohtaka-Maruyama, C. et al. Synaptic transmission from subplate neurons controls radial migration of neocortical neurons. Science 360, 313-317 (2018).

74. Tabata, H. \& Nakajima, K. Neurons tend to stop migration and differentiate along the cortical internal plexiform zones in the Reelin signal-deficient mice. J. Neurosci. Res. 69, 723-730 (2002).

75. Rice, D. S. et al. Disabled-1 acts downstream of Reelin in a signaling pathway that controls laminar organization in the mammalian brain. Development 125, 3719-3729 (1998).

76. Jossin, Y. \& Cooper, J. A. Reelin, Rap 1 and N-cadherin orient the migration of multipolar neurons in the developing neocortex. Nat. Neurosci. 14, 697-703 (2011)

This work shows that reelin released by CR cells controls the repolarization of multipolar neurons in the SVZ and/or intermediate zone, allowing them to initiate locomotion on RG fibres.

77. Ogawa, M. et al. The reeler gene-associated antigen on Cajal-Retzius neurons is a crucial molecule for laminar organization of cortical neurons. Neuron 14 899-912 (1995)

78. Yoshida, M., Assimacopoulos, S., Jones, K. R. $\&$ Grove, E. A. Massive loss of Cajal-Retzius cells does not disrupt neocortical layer order. Development 133 , 537-545 (2006)

79. Kupferman, J. V. et al. Reelin signaling specifies the molecular identity of the pyramidal neuron distal dendritic compartment. Cell 158, 1335-1347 (2014)

80. Hevner, R. F., Daza, R. A., Englund, C., Kohtz, J. \& Fink, A. Postnatal shifts of interneuron position in the neocortex of normal and reeler mice: evidence for inward radial migration. Neuroscience 124, 605-618 (2004).

81. Anstotz, M. et al. Morphology, input-output relations and synaptic connectivity of Cajal-Retzius cells in layer 1 of the developing neocortex of CXCR4-EGFP mice. Brain Struct. Funct. 219, 2119-2139 (2014).

82. Opris, I., Chang, S. \& Noga, B. R. What is the evidence for inter-laminar integration in a prefrontal cortical minicolumn? Front Neuroanat 11, 116 (2017).

83. Rakic, P. Radial versus tangential migration of neuronal clones in the developing cerebral cortex. Proc. Natl Acad. Sci. USA 92, 11323-11327 (1995).

84. Dimidschstein, J. et al. Ephrin-B1 controls the columnar distribution of cortical pyramidal neurons by restricting their tangential migration. Neuron $\mathbf{7 9}$, 1123-1135 (2013)

85. Lodato, S. et al. Excitatory projection neuron subtypes control the distribution of local inhibitory interneurons in the cerebral cortex. Neuron 69 763-779 (2011)

86. Xue, M., Atallah, B. V. \& Scanziani, M. Equalizing excitation-inhibition ratios across visual cortical neurons. Nature 511, 596-600 (2014).

87. Cobos, I. et al. Mice lacking DIx 1 show subtype-specific loss of interneurons, reduced inhibition and epilepsy. Nat. Neurosci. 8, 1059-1068 (2005).

88. Marin, O. Interneuron dysfunction in psychiatric disorders. Nat. Rev. Neurosci. 13, 107-120 (2012)

89. Southwell, D. G. et al. Intrinsically determined cell death of developing cortical interneurons. Nature 491, 109-113 (2012)

90. Denaxa, M. et al. Modulation of apoptosis controls inhibitory interneuron number in the cortex. Cell Rep. 22, 1710-1721 (2018)

91. Wong, F. K. et al. Pyramidal cell regulation of interneuron survival sculpts cortical networks. Nature 557, 668-673 (2018).

92. Sessa, A. et al. Tbr2-positive intermediate (basal) neuronal progenitors safeguard cerebral cortex expansion by controlling amplification of pallial glutamatergic neurons and attraction of subpallial GABAergic interneurons. Genes Dev. 24, 1816-1826 (2010)

93. Voronova, A. et al. Migrating interneurons secrete fractalkine to promote oligodendrocyte formation in the developing mammalian brain. Neuron 94 500-516 (2017)

94. Seuntjens, E. et al. Sip 1 regulates sequential fate decisions by feedback signaling from postmitotic neurons to progenitors. Nat. Neurosci. 12 , 1373-1380 (2009)

This study unravels a feedback regulatory loop from newborn postmitotic PNs to their progenitors in order to control the sequential generation of PNs dedicated to distinct layers.

95. Wang, W. et al. Feedback regulation of apical progenitor fate by immature neurons through
Wnt7-Celsr3-Fzd3 signalling. Nat. Commun. 7, 10936 (2016).

96. Fantin, A. et al. Tissue macrophages act as cellular chaperones for vascular anastomosis downstream of VEGF-mediated endothelial tip cell induction. Blood 116, 829-840 (2010).

97. Goldmann, T. et al. Origin, fate and dynamics of macrophages at central nervous system interfaces. Nat. Immunol. 17, 797-805 (2016).

98. Rymo, S. F. et al. A two-way communication between microglial cells and angiogenic sprouts regulates angiogenesis in aortic ring cultures. PLOS ONE 6 e15846 (2011)

99. Shigemoto-Mogami, Y., Hoshikawa, K., Goldman, J. E., Sekino, Y. \& Sato, K. Microglia enhance neurogenesis and oligodendrogenesis in the early postnatal subventricular zone. J. Neurosci. 34, 2231-2243 (2014).

100. Balia, M., Benamer, N. \& Angulo, M. C. A specific GABAergic synapse onto oligodendrocyte precursors does not regulate cortical oligodendrogenesis. Glia 65, 1821-1832 (2017)

101. Orduz, D. et al. Interneurons and oligodendrocyte progenitors form a structured synaptic network in the developing neocortex. eLife 4, e06953 (2015).

102. Tielens, S., Godin, J. D. \& Nguyen, L. Real-time recordings of migrating cortical neurons from GFP and Cre recombinase expressing mice. Curr. Protoc. Neurosci. 74, 3.29.1-3.29.23 (2016).

103. Gao, X. et al. Artificial microniche array with spatially structured biochemical cues. Methods $\mathrm{Mol}$. Biol. 1771, 55-66 (2018)

104. Decock, J., Schlenk, M. \& Salmon, J. B. In situ photo-patterning of pressure-resistant hydrogel membranes with controlled permeabilities in PEGDA microfluidic channels. Lab. Chip 18, 1075-1083 (2018).

105. Lancaster, M. A. et al. Cerebral organoids model human brain development and microcephaly. Nature 501, 373-379 (2013).

106. Birey, F. et al. Assembly of functionally integrated human forebrain spheroids. Nature 545, 54-59 (2017).

This works describes a novel method consisting of fusing human patterned organoids to study the migration of interneurons invading pallial-like structures.

107. Xiang, Y. et al. Fusion of regionally specified hPSC-derived organoids models human brain development and interneuron migration. Cell Stem Cell 21, 383-398 (2017).

108. Bagley, J. A., Reumann, D., Bian, S., Levi-Strauss, J. \& Knoblich, J. A. Fused cerebral organoids model interactions between brain regions. Nat. Methods 14 743-751 (2017)

109. Takahashi, T., Nowakowski, R. S. \& Caviness, V. S. Jr. The leaving or $\mathrm{Q}$ fraction of the murine cerebral proliferative epithelium: a general model of neocortical neuronogenesis. J. Neurosci. 16 6183-6196 (1996).

110. Gohlke, J. M., Griffith, W. C. \& Faustman, E. M Computational models of neocortical neuronogenesis and programmed cell death in the developing mouse, monkey, and human. Cereb.Cortex 17, 2433-2442 (2007).

111. Cahalane, D. J., Charvet, C. J. \& Finlay, B. L. Modeling local and cross-species neuron number variations in the cerebral cortex as arising from a common mechanism. Proc. Natl Acad. Sci. USA 111, 17642-17647 (2014)

112. Hsu, L. C. et al. Lhx2 regulates the timing of beta-catenin-dependent cortical neurogenesis. Proc. Natl Acad. Sci. USA 112, 12199-12204 (2015).

113. Freret-Hodara, B. et al. Enhanced abventricular proliferation compensates cell death in the embryonic cerebral cortex. Cereb. Cortex 27, 4701-4718 (2017).

114. Setty, Y. et al. How neurons migrate: a dynamic in-silico model of neuronal migration in the developing cortex. BMC Syst. Biol. 5, 154 (2011).

115. Strauss, K. A. et al. Recessive symptomatic focal epilepsy and mutant contactin-associated protein-like 2. N. Engl. J. Med. 354, 1370-1377 (2006).

116. Penagarikano, O. et al. Absence of CNTNAP2 leads to epilepsy, neuronal migration abnormalities, and core autism-related deficits. Cell 147, 235-246 (2011).

117. Selimbeyoglu, A. et al. Modulation of prefrontal cortex excitation/inhibition balance rescues social behavior in CNTNAP2-deficient mice. Sci. Trans/ Med. 9 , eaah6733 (2017).
118. Vogt, D. et al. Mouse Cntnap2 and human CNTNAP2 ASD alleles cell autonomously regulate $\mathrm{PV}+$ cortical interneurons. Cereb. Cortex 28, 3868-3879 (2017).

119. Penagarikano, O., Mulle, J. G. \& Warren, S. T. The pathophysiology of fragile x syndrome. Annu. Rev. Genomics Hum. Genet. 8, 109-129 (2007).

120. La Fata, G. et al. FMRP regulates multipolar to bipolar transition affecting neuronal migration and cortical circuitry. Nat. Neurosci. 17, 1693-1700 (2014).

121. Selby, L., Zhang, C. \& Sun, Q. Q. Major defects in neocortical CABAergic inhibitory circuits in mice lacking the fragile $\mathrm{X}$ mental retardation protein Neurosci. Lett. 412, 227-232 (2007).

122. Manent, J. B., Wang, Y., Chang, Y., Paramasivam, M. $\delta$ LoTurco, J. J. Dcx reexpression reduces subcortical band heterotopia and seizure threshold in an animal model of neuronal migration disorder. Nat. Med. 15 , 84-90 (2009)

This study shows that re-expression of DCX in postnatal rats suffering from its embryonic depletion partially rescues radial migration defects. This work thus highlights a postnatal time window for cerebral cortex repair.

123. Lancaster, M. A. et al. Guided self-organization and cortical plate formation in human brain organoids. Nat. Biotechnol. 35, 659-666 (2017).

124. McKee, C. \& Chaudhry, G. R. Advances and challenges in stem cell culture. Colloids Surf. B Biointerfaces 159 62-77 (2017)

125. Markram, H. et al. Reconstruction and simulation of neocortical microcircuitry. Cell 163, 456-492 (2015).

126. Rakic, P. Evolution of the neocortex: a perspective from developmental biology. Nat. Rev. Neurosci. 10, 724-735 (2009).

127. Geschwind, D. H. \& Rakic, P. Cortical evolution judge the brain by its cover. Neuron $\mathbf{8 0}, 633-647$ (2013).

128. Walsh, C. \& Cepko, C. L. Clonal dispersion in proliferative layers of developing cerebral cortex. Nature 362, 632-635 (1993).

129. Metin, C. et al. Conserved pattern of tangential neuronal migration during forebrain development Development 134, 2815-2827 (2007)

130. Tanaka, D. H. \& Nakajima, K. GABAergic interneuron migration and the evolution of the neocortex. Dev. Growth Differ. 54, 366-372 (2012).

131. Ma, T. et al. Subcortical origins of human and monkey neocortical interneurons. Nat. Neurosci. 16, 1588-1597 (2013).

132. Paredes, M. F. et al. Extensive migration of young neurons into the infant human frontal lobe. Science 354, aaf7073 (2016)

133. Letinic, K. \& Rakic, P. Telencephalic origin of human thalamic GABAergic neurons. Nat. Neurosci. 4 931-936 (2001).

134. Rao, Y. \& Wu, J. Y. Neuronal migration and the evolution of the human brain. Nat. Neurosci. 4 860-862 (2001)

135. Bielle, F. et al. Slit2 activity in the migration of guidepost neurons shapes thalamic projections during development and evolution. Neuron 69, 1085-1098 (2011). This study shows that ventral migration of guidepost cells opens the path for TC axons to reach the developing cerebral cortex.

136. Lopez-Bendito, G. et al. Tangential neuronal migration controls axon guidance: a role for neuregulin-1 in thalamocortical axon navigation. Cell 125, 127-142 (2006).

137. Barros, C. S., Franco, S. J \& Muller U. Extracellular matrix: functions in the nervous system Cold Spring Harb. Perspect. Biol. 3, a005108 (2011).

138. Erickson, A. C. \& Couchman, J. R. Still more complexity in mammalian basement membranes. J. Histochem. Cytochem. 48, 1291-1306 (2000).

139. Timpl, R. \& Brown, J. C. Supramolecular assembly of basement membranes. Bioessays 18, 123-132 (1996).

140. Beltran-Valero de Bernabe, D. et al. Mutations in the O-mannosyltransferase gene POMT1 give rise to the severe neuronal migration disorder Walker-Warburg syndrome. Am. J. Hum. Genet. 71, 1033-1043 (2002).

141. Chen, Z. L., Haegeli, V., Yu, H. \& Strickland, S. Cortical deficiency of laminin gamma 1 impairs the AKT/GSK3 beta signaling pathway and leads to defects in neurite outgrowth and neuronal migration. Dev. Biol. 327, 158-168 (2009)

142. Georges-Labouesse, E., Mark, M., Messaddeq, N. \& 
cortical and retinal lamination. Curr. Biol. 8, 983-986 (1998).

143. Radakovits, R., Barros, C. S., Belvindrah, R., Patton, B. $\&$ Muller, U. Regulation of radial glial survival by signals from the meninges. J. Neurosci. 29 , 7694-7705 (2009).

144. Lathia, J. D. et al. Patterns of laminins and integrins in the embryonic ventricular zone of the CNS. J. Comp. Neurol. 505, 630-643 (2007).

145. Loulier, K. et al. beta1 integrin maintains integrity of the embryonic neocortical stem cell niche. PLOS Biol. 7, e1000176 (2009).

146. Stanco, A. et al. Netrin-1-alpha3beta 1 integrin interactions regulate the migration of interneurons through the cortical marginal zone. Proc. Natl Acad. Sci. USA 106, 7595-7600 (2009).

147. Zimmer, G. et al. Chondroitin sulfate acts in concert with semaphorin $3 \mathrm{~A}$ to guide tangential migration of cortical interneurons in the ventral telencephalon. Cereb. Cortex 20, 2411-2422 (2010).

148. Kornack, D. R. \& Rakic, P. Radial and horizontal deployment of clonally related cells in the primate neocortex: relationship to distinct mitotic lineages. Neuron 15, 311-321 (1995)

149. Luskin, M. B., Pearlman, A. L. \& Sanes, J. R. Cell lineage in the cerebral cortex of the mouse studied in vivo and in vitro with a recombinant retrovirus. Neuron 1, 635-647 (1988).
150. Evrony, G. D. et al. Cell lineage analysis in human brain using endogenous retroelements. Neuron 85 49-59 (2015)

151. Garcia-Moreno, F., Vasistha, N. A., Begbie, J. \& Molnar, Z. CLoNe is a new method to target single progenitors and study their progeny in mouse and chick. Development 141, 1589-1598 (2014).

152. Livet, J. et al. Transgenic strategies for combinatorial expression of fluorescent proteins in the nervous system. Nature 450, 56-62 (2007).

153. Loulier, K. et al. Multiplex cell and lineage tracking with combinatorial labels. Neuron 81, 505-520 (2014).

154. Hippenmeyer, S. et al. Genetic mosaic dissection of Lis1 and Ndel 1 in neuronal migration. Neuron 68 , 695-709 (2010).

155. Beattie, R. et al. Mosaic analysis with double markers reveals distinct sequential functions of Lgl 1 in neural stem cells. Neuron 94, 517-533 (2017).

156. Brown, K. N. et al. Clonal production and organization of inhibitory interneurons in the neocortex. Science 334, 480-486 (2011).

157. Ciceri, G. et al. Lineage-specific laminar organization of cortical GABAergic interneurons. Nat. Neurosci. 16 1199-1210 (2013).

158. Mayer, C. et al. Clonally related forebrain interneurons disperse broadly across both functional areas and structural boundaries. Neuron 87, 989-998 (2015).

\section{Acknowledgements}

The authors thank M. H. Adhikari for critical reading. C.G.S. is paid by F.R.S.-FNRS (EOS 0019118 F-RG36). E.P. is paid by the IAP-VII network P7/20 and a Federation of European Biochemical Societies (FEBS) short-term fellowship. L.N. is a senior research associate of the F.R.S-FNRS at the University of Liège. His research is funded by F.R.S.-FNRS (EOS 0019118F-RG36, CDR J.0028.18 and PDR T.0073.15), the Fonds Léon Fredericq, the Fondation Médicale Reine Elisabeth, the Fondation Simone et Pierre Clerdent, the Belgian Science Policy (IAP-VII network P7/20), the ARC (ARC11/16-01), the ERANET Neuron STEM-MCD and the ERANET Neuron NEUROTALK.

\section{Author contributions}

All authors contributed equally to all aspects of the manuscript.

\section{Competing interests}

The authors declare no competing interests.

\section{Publisher's note}

Springer Nature remains neutral with regard to jurisdictional claims in published maps and institutional affiliations.

\section{Reviewer information}

Nature Reviews Neuroscience thanks S. Pleasure and K. Nagata for their contribution to the peer review of this work. 